\title{
Passivation Effects of Aluminum on Polycrystalline Silicon Thin-Film Transistor With Metal-Replaced Junctions
}

\author{
Dongli Zhang and Man Wong, Senior Member, IEEE
}

\begin{abstract}
Aluminum was detected in the channel of a thin-film transistor after its replacement of the polycrystalline silicon source and drain junctions. The resulting transistor exhibits enhanced field-effect mobility, steeper slope of the pseudosubthreshold region, reduced turn-on voltage extrapolated from the linear regime of operation, higher ON-state current, and improved immunity against short-channel effects. These improvements are consistent with a measured reduction in the density of trap states. The reduction can be attributed to the presence of aluminum in the channel.
\end{abstract}

Index Terms-Aluminum, passivation, polycrystalline silicon, thin-film transistor (TFT).

\section{INTRODUCTION}

$\mathbf{E}$ XHIBITING higher field-effect mobility than the amorphous silicon thin-film transistors (TFTs), the polycrystalline silicon (poly-Si) TFTs are being applied to realizing systems-on-glass [1], such as the self-scanned active-matrix flat-panel displays [2]. Replacement of poly-Si with aluminum (Al) has been applied to the formation of TFTs with metalreplaced junctions (MERJs) [3], [4]. Although the poly-Si active islands of such TFTs are initially undoped, the channel regions immediately beyond the replacement fronts are subsequently doped with $\mathrm{Al}$, which is an acceptor in Si [5]. Consequently, the detrimental effects of parasitic resistance are largely eliminated by the low resistance of the resulting metallic junctions and the ohmic contacts. However, little is known if there is diffusion of $\mathrm{Al}$ farther beyond the replacement fronts; and if there is, what effects $\mathrm{Al}$ would have on the characteristics of the TFTs.

A comparison was made of the intrinsic characteristics of TFTs with and without MERJs. The MERJ TFT was found to exhibit an enhanced field-effect mobility, steeper slope $S_{p}$ of the pseudosubthreshold region [6], reduced turn-on voltage $V_{\text {on }}$ [7] extrapolated from the linear regime of operation, higher ON-state current, and improved immunity against short-channel effects. Consistent with the improvements, a reduced density of trap states in the channel region of a MERJ TFT was extracted.

Manuscript received November 8, 2006; revised December 2, 2006. This work was supported by a grant from the Research Grants Council of the Hong Kong Special Administrative Region. The review of this letter was arranged by Editor Y. Taur.

The authors are with the Department of Electronic and Computer Engineering, The Hong Kong University of Science and Technology, Kowloon, Hong Kong (e-mail: eemwong@ee.ust.hk).

Digital Object Identifier 10.1109/LED.2006.889506
This reduction is attributed to the passivation of the traps by $\mathrm{Al}$; a small amount of which is detected using time-of-flight secondary ion-mass spectrometry (TOF-SIMS) in the channel region far beyond the replacement fronts.

\section{DEVICE FABRICATION}

100-mm diameter, (100)-oriented n-type silicon wafers covered with 500-nm thermally grown silicon dioxide were used as the starting substrates. Conventional nickel-based metalinduced crystallization [8] at $550{ }^{\circ} \mathrm{C}$ was performed to obtain $35-\mathrm{nm}$ thick poly-Si active islands. Following the sequential low-pressure chemical vapor depositions of $90-\mathrm{nm}$ lowtemperature oxide (LTO) at $450{ }^{\circ} \mathrm{C}$ as the gate dielectric and 220-nm poly-Si at $620{ }^{\circ} \mathrm{C}$, the latter was patterned to form the gate electrodes. Self-aligned gate, source, and drain doping was accomplished by $4 \times 10^{15} \mathrm{~cm}^{-2}$ boron implantation at an energy of $45 \mathrm{keV}$. The implanted dopant was activated at $620^{\circ} \mathrm{C}$ for $3 \mathrm{~h}$ in atmospheric pressure nitrogen.

Subsequently, 200-nm LTO isolation layer was deposited, and through which, the replacement windows were opened before $2-\mu \mathrm{m} \mathrm{Al}-1 \% \mathrm{Si}$ alloy, and 300-nm titanium (Ti) was sequentially deposited by sputtering. The Ti was selectively removed in concentrated hydrofluoric acid from some of the active islands. On those islands with the top Ti retained, the boron-doped poly-Si at the source and drain regions was completely replaced by $\mathrm{Al}$ after a heat treatment at $400{ }^{\circ} \mathrm{C}$ for $2 \mathrm{~h}$ in atmospheric pressure nitrogen. No replacement was observed on the islands with the Ti removed. On these islands were built the "conventional" TFTs. All remaining Ti was subsequently removed in hydrofluoric acid before the $\mathrm{Al}$ layer was patterned to form the interconnections.

After the removal of the covering poly-Si gate and gate oxide, TOF-SIMS using $25-\mathrm{keV} \mathrm{Ga}^{+}$ion was applied to detect $\mathrm{Al}$ in the channel regions of the MERJ and the conventional TFTs. HP 4156 Semiconductor Parameter Analyzer was used to obtain the current-voltage characteristics.

\section{RESUltS AND DisCUSSION}

Typical drain-current $\left(I_{d}\right)$ versus terminal gate-to-source voltage $\left(V_{\mathrm{gs}}\right)$ transfer characteristics of the MERJ and conventional TFTs with channel width $W$ of $10 \mu \mathrm{m}$ and length $L$ of $25 \mu \mathrm{m}$ are shown in Fig. 1. The leakage current $\left(I_{\text {off }}\right)$ is defined to be the minimum $I_{d}$ at a terminal drain-to-source voltage 


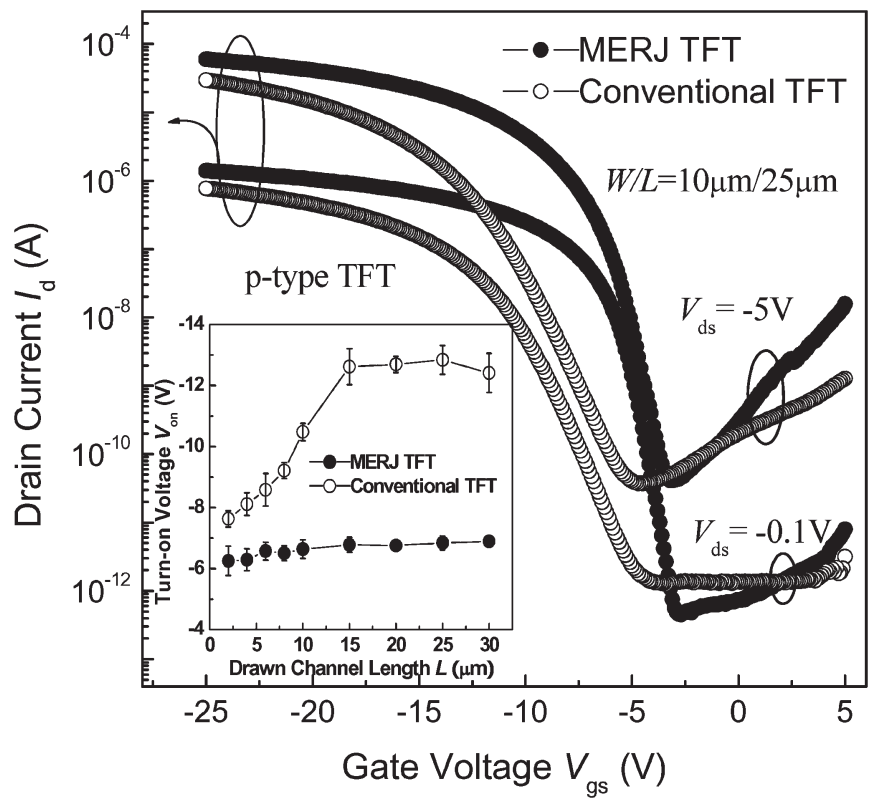

Fig. 1. $I_{d}-V_{\mathrm{gs}}$ characteristics for p-type MERJ and conventional TFTs at $V_{\mathrm{gs}}=-0.1 \mathrm{~V}$ and $-5 \mathrm{~V}$. Shown in the inset is the dependence of $V_{\mathrm{on}}$ on $L$ for both the MERJ and conventional TFTs. $W$ is $10 \mu \mathrm{m}$ for all of the TFTs.

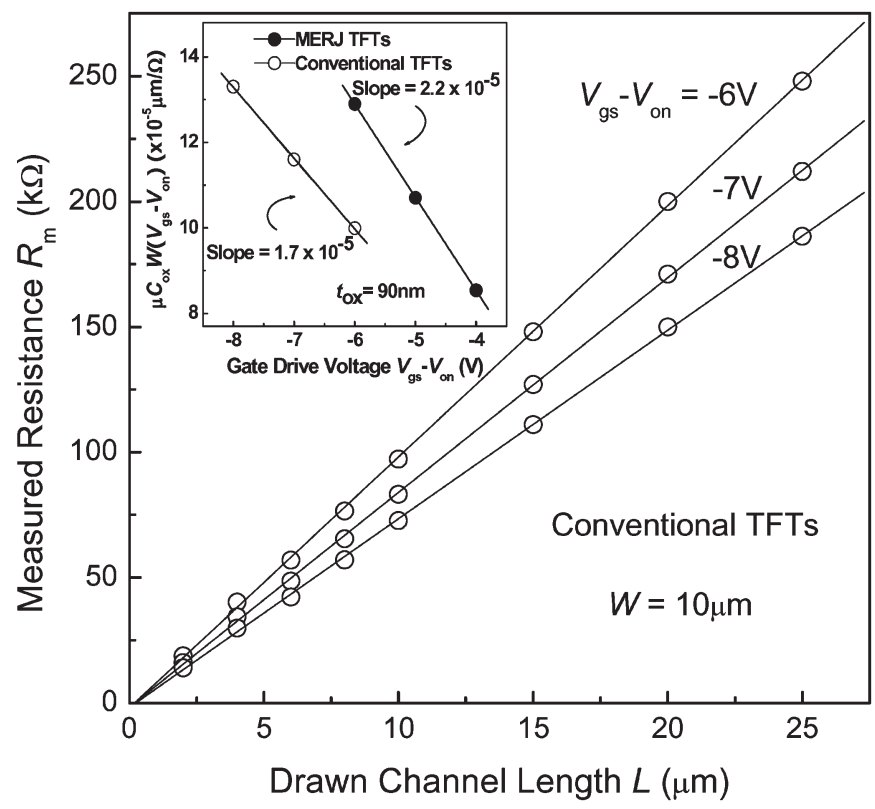

Fig. 2. Measured $R_{m} \equiv\left(V_{\mathrm{ds}} / I_{d}\right)$ versus $L$ of a series of conventional TFTs at different values of the gate drive $\left(V_{\mathrm{gs}}-V_{\mathrm{on}}\right)$. The dependence of the slopes of the lines on $V_{\mathrm{gs}}-V_{\mathrm{on}}$, together with similar data derived from the MERJ TFTs, is shown in the inset. $W$ is $10 \mu \mathrm{m}$ for all of the TFTs.

$V_{\mathrm{ds}}=-5 \mathrm{~V}$, and the ON-state current $\left(I_{\mathrm{on}}\right)$ is $I_{d}$ at $V_{\mathrm{ds}}=-5 \mathrm{~V}$ and $V_{\mathrm{gs}}=-25 \mathrm{~V}$.

Fig. 2 is generated from the transfer characteristics of a series of conventional TFTs at $V_{\mathrm{ds}}=-0.05 \mathrm{~V}$. Eliminating the effects of parasitic source/drain resistance using the procedure outlined in [9], one extracts an "intrinsic" $\mu_{\mathrm{FE}}$ of $\sim 44.6 \mathrm{~cm}^{2} / \mathrm{V} \cdot \mathrm{s}$ for the linear regime of operation. Similarly, a $\mu_{\mathrm{FE}}$ of $57.3 \mathrm{~cm}^{2} / \mathrm{V} \cdot \mathrm{s}$ is extracted for the MERJ TFTs. $V_{\text {on }}$ and $S_{p}$ are extracted at $V_{\mathrm{ds}}=-0.1 \mathrm{~V}$. These and other characteristics of the MERJ and conventional TFTs are sum-
TABLE I

COMPARISON OF THE ElECTRICAL CHARACTERISTICS OF P-TYPE MERJ and Conventional TFTs. THE Respective DraWn CHANNEL WIDTH $W$ AND LENGTH $L$ ARE 10 AND $25 \mu \mathrm{m}$

\begin{tabular}{|c|c||c|}
\hline & MERJ TFT & Conventional TFT \\
\hline \hline$\mu_{\mathrm{FE}}\left(\mathrm{cm}^{2} / \mathrm{Vs}\right)$ & $55 \pm 1.5$ & $44 \pm 1.2$ \\
\hline$V_{\mathrm{on}}(\mathrm{V})$ & $-6.9 \pm 0.2$ & $-12.9 \pm 0.4$ \\
\hline$S_{\mathrm{p}}(\mathrm{V} /$ decade $)$ & $0.7 \pm 0.1$ & $1.5 \pm 0.1$ \\
\hline$I_{\text {on }}(\mu \mathrm{A} / \mu \mathrm{m})$ & $5.9 \pm 0.1$ & $3.0 \pm 0.2$ \\
\hline$I_{\text {off }}(\mathrm{pA} / \mu \mathrm{m})$ & $6.2 \pm 1.6$ & $2.6 \pm 1.0$ \\
\hline$R_{\mathrm{sd}}(\Omega)$ & 1.5 & 641 \\
\hline
\end{tabular}

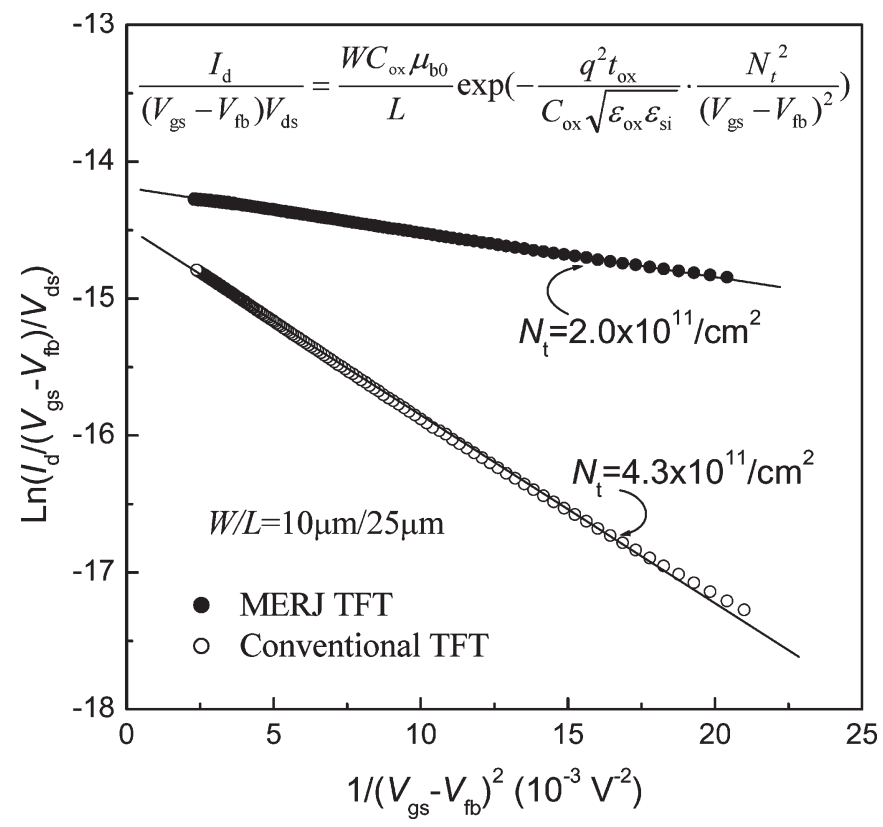

Fig. 3. Plots of $\ln \left(I_{d} /\left(V_{\mathrm{gs}}-V_{\mathrm{fb}}\right) V_{\mathrm{ds}}\right)$ versus $1 /\left(V_{\mathrm{gs}}-V_{\mathrm{fb}}\right)^{2}$ for the conventional and MERJ TFTs. $N_{t}$ is the trap density, $\varepsilon_{\mathrm{Si}}$ is the silicon dielectric constant, $\varepsilon_{\mathrm{ox}}$ is the silicon dioxide dielectric constant, $t_{\mathrm{ox}}$ is the gate oxide thickness, $q$ is the electron charge, $\mu_{b 0}$ is the mobility constant, $C_{\text {ox }}$ is the gate oxide capacitance per unit area, and $V_{\mathrm{fb}}$ is the flatband voltage. The circles are measured data and the solid lines are linearly fitting curves to the data.

marized in Table I, showing respective $S_{p}$ of $0.7 \mathrm{~V} / \mathrm{dec}$ and $1.5 \mathrm{~V} / \mathrm{dec}$, and respective $V_{\text {on }}$ of $-7 \mathrm{~V}$ and $-13 \mathrm{~V}$. The parasitic junction resistance $R_{\mathrm{sd}}$ of the MERJ TFT is more than two orders of magnitude smaller than that of the conventional TFT. The reduction in $V_{\text {on }}$, when combined with a concurrent $\sim 25 \%$ increase in $\mu_{\mathrm{FE}}$, leads to an almost doubling of the $I_{\mathrm{on}}$ of a MERJ TFT.

The short-channel effects in TFTs are typically manifested by a reduction in $V_{\text {on }}$ with decreasing $L$, as shown in the inset of Fig. 1. Such behavior is quite obvious for the conventional TFTs, with $V_{\text {on }}$ dropping by $\sim 5 \mathrm{~V}$ from $L=15 \mu \mathrm{m}$ to $L=$ $4 \mu \mathrm{m}$. On the contrary, the $V_{\text {on }}$ reduction is rather subtle for the MERJ TFTs within the range of $L$ between 4 and $30 \mu \mathrm{m}$.

It is known that the characteristics of poly-Si TFT, including the short-channel effects [10], are heavily influenced by the density of trap states. Extracted from Fig. 3 using a technique proposed in [11], the respective densities for the conventional and MERJ TFTs are $4.3 \times 10^{11}$ and $2.0 \times 10^{11} \mathrm{~cm}^{-2}$. After the removal of the covering poly-Si gate and gate oxide, $\mathrm{Al}$ was 


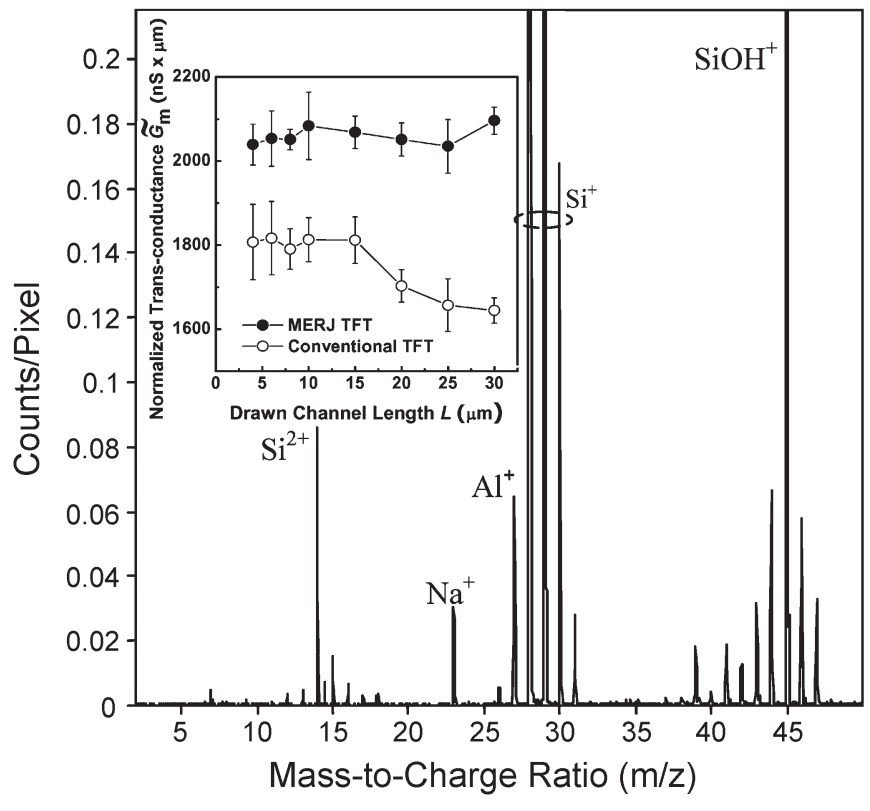

Fig. 4. Positive ion TOF-SIMS spectrum taken near the middle of the channel of a MERJ TFT with $L=25 \mu \mathrm{m}$. Shown in the inset is the normalized transconductance $\widetilde{G}_{m} \equiv G_{m} L$ versus $L$ for the conventional and MERJ TFTs. $W$ is $10 \mu \mathrm{m}$ for all of the TFTs.

detected (Fig. 4) using TOF-SIMS in the channel regions of the MERJ TFTs, but not in those of the conventional TFTs. Since the two kinds of TFTs were fabricated in parallel, it is reasonable to attribute the observed reduction in the density of traps in MERJ TFTs to the presence and passivation effects of Al.

Although the characteristics of TFTs with $L=25 \mu \mathrm{m}$ are reported in detail, the improvements are seen for TFTs with other values of $L$. This is clear from the comparison of the maximum normalized transconductance $\tilde{G}_{m} \equiv G_{m} L$ given in the inset of Fig. 4, where $G_{m}$ is the maximum transconductance at $V_{\mathrm{ds}}=-0.1 \mathrm{~V}$. Although the $G_{m}$ of the conventional TFT increases with decreasing $L$, passivation-induced improvements are evident in all the TFTs within the range of $L$ investigated (insets of Figs. 1 and 4). It is clear from the improvement of the TFT with $L=30 \mu \mathrm{m}$ that the diffusivity of Al in the polycrystalline silicon formed using metal-induced crystallization is quite high and gives rise to a diffusion length of at least $15 \mu \mathrm{m}$ at the end of the $400{ }^{\circ} \mathrm{C}, 2-\mathrm{h}$ replacement heat treatment.

\section{CONCLUSION}

A comparison was made of the characteristics of TFTs with and without MERJs. It was found that the transistors with such junctions exhibited an enhanced field-effect mobility, steeper slope of the pseudosubthreshold region, reduced turnon voltage extrapolated from the linear regime of operation, higher ON-state current, and improved immunity against shortchannel effects. Aluminum was detected in the channel of a transistor after its replacement of the polycrystalline silicon source and drain junctions. These improvements are consistent with a measured reduction in the density of trap states in the channel. The reduction is attributed to the passivation of such states by aluminum.

\section{REFERENCES}

[1] F. Okumura, "Development trend of system-on-glass LCD for mobile applications," in Proc. IDMC, 2005, pp. 311-314.

[2] C. Y. Wu, Z. G. Meng, S. Z. Xiong, M. Wong, and H. S. Kwok, "Application of metal induced unilaterally crystallized polycrystalline silicon thin-film transistor technology to system-on-glass display," J. NonCryst. Solids, vol. 352, no. 9-20, pp. 1741-1744, Jun. 2006.

[3] D. L. Zhang and M. Wong, "Metal-replaced junction (MERJ) for reducing the junction parasitic resistance of a thin-film transistor," IEEE Electron Device Lett., vol. 27, no. 4, pp. 269-271, Apr. 2006.

[4] —, "Three-mask polycrystalline silicon thin-film transistor with metallic gate and junctions," IEEE Electron Device Lett., vol. 27, no. 7, pp. 564-566, Jul. 2006.

[5] B. Y. Tsaur, G. W. Turner, and J. C. C. Fan, "Efficient Si solar cells by low-temperature solid phase epitaxy," Appl. Phys. Lett., vol. 39, no. 9, pp. 749-751, Nov. 1981.

[6] T. S. Li and P. S. Lin, "On the pseudo sub-threshold characteristics of polycrystalline silicon thin-film transistors with large grain size," IEEE Electron Device Lett., vol. 14, no. 5, pp. 240-242, May 1993.

[7] M. D. Jacunski, M. S. Shur, and M. Hack, "Threshold voltage, fieldeffect mobility and gate-to-channel capacitance in polysilicon TFTs," IEEE Trans. Electron Devices, vol. 43, no. 9, pp. 1433-1440, Sep. 1996.

[8] Z. G. Meng, S. Y. Zhao, C. Y. Wu, B. Zhang, M. Wong, and H.-S. Kwok, "Polycrystalline silicon films and thin-film transistors using solutionbased metal-induced crystallization," J. Display Technol., vol. 2, no. 3, pp. 265-273, Sep. 2006.

[9] D. L. Zhang and M. Wong, "Effects of trace nickel on the growth kinetics and the electrical characteristics of metal-induced laterally crystallized polycrystalline silicon and devices," J. Soc. Inf. Display, vol. 13, no. 10, pp. 815-822, Oct. 2005.

[10] A. G. Lewis, T. Y. Huang, I. W. Wu, R. H. Bruce, and A. Chiang, "Physical mechanisms for short channel effects in polysilicon thin film transistors," in IEDM Tech. Dig., 1989, pp. 349-352.

[11] R. E. Proano, R. S. Misage, and D. G. Ast, "Development and electrical properties of undoped polycrystalline silicon thin-film transistors," IEEE Trans. Electron Devices, vol. 36, no. 9, pp. 1915-1922, Sep. 1989. 